\title{
His bundle recordings in bradycardia-dependent AV block induced by premature beats
}

\author{
Agustin Castellanos, Shaik A. Khuddus, Leonard S. Sommer, Ruey J. Sung, and Robert J. \\ Myerburg \\ From the Division of Cardiology, Department of Medicine, University of Miami School of Medicine and The \\ fackson Memorial Hospital, Miami, Florida, U.S.A.
}

Symptomatic bradycardia-dependent atrioventricular block occurred in a patient with right bundle-branch block, left anterior hemiblock, and prolonged HV interval. The arrhythmia, triggered by spontaneous or induced premature beats, appeared when the post-extrasystolic PP and HH intervals increased to a critical value. Reinitiation of atrioventricular conduction required the presence of ventricular escapes. Bradycardiadependent atrioventricular block was related to either an enhanced or slightly rising slope of diastolic depolarization, or to a decrease in membrane responsiveness. The patient also, most probably, had tachycardiadependent atrioventricular block.

Both types of conduction disturbance occurred in the same part of the intraventricular conducting system, either in the low His bundle or left bundle-branch or its posteroinferior division. It is suggested that the electrophysiological study of cases with prolonged HV intervals should include procedures which can expose bradycardia-dependent atrioventricular block.

Although the genesis of rate-related bundle-branch block has been a subject of considerable debate and speculation (see recent review by Elizari et al., 1974 and El-Sherif et al., 1974), there have been few published cases of symptomatic bradycardia-dependent atrioventricular block in which His bundle electrograms were recorded (Coumel et al., 1971; Castellanos et al., r974; Goodfriend and Barold, 1974). It is therefore justifiable to present the findings observed in one patient with this form of paroxysmal atrioventricular block and to speculate on its electrophysiological mechanisms and possible clinical relevance.

\section{Case report}

A 66-year-old Cuban man, brought to the emergency room because of a syncopal attack, had been asymptomatic until the day of admission. Though there was no history of angina pectoris or myocardial infarction the twelve-lead electrocardiogram showed changes compatible with anterior (and perhaps inferior) wall myocardial infarction of undetermined age (Fig. I). Right bundle-branch block and left anterior hemiblock were also present. On arrival at the coronary care unit he experienced a dizzy spell caused by an episode of paroxysmal atrioventricular block (Fig. 2).

Two additional similar episodes occurred while Received 16 August 1974. awaiting for pacemaker insertion. In all instances the conduction 'disturbance was triggered by premature beats which could have been atrioventricular junctional or ventricular, with retrograde conduction to the atria. At the beginning of Fig. 2 the intervals between sinus $P$ waves measured $720 \mathrm{~ms}$. The fifth, ectopic, QRS complex was followed by a retrograde atrial deflection $\left(\mathrm{P}^{-}\right)$which caused a prolongation of the subsequent $\mathbf{P}^{-}-$ $P$ cycle to $800 \mathrm{~ms}$. This increase in atrial cycle length was probably responsible for the initiation of a run of paroxysmal atrioventricular block which was terminated by an escape beat. Thereafter I:I atrioventricular conduction was resumed.

The mechanisms of the conduction disturbance were not apparent at that moment, but it was thought that pacemaker therapy was justified because the arrhythmia was symptomatic. The situation was, therefore, explained to the patient and wife. Consent was obtained for pacemaker insertion as well as for performing intracardiac electrophysiological studies (Castillo and Castellanos, I97I; Castellanos et al., 1974).

His bundle electrograms, recorded as previously described (Castillo and Castellanos, 197I; Castellanos et al; 1974), showed that during sinus rhythm (Fig. 3) the $\mathrm{HV}$ interval measured $80 \mathrm{~ms}$ (upper limits of normal in our department: $55 \mathrm{~ms}$ ). In the presence of right bundle branch block and left anterior hemiblock this indicates the existence of a conduction delay in the low His bundle (below the site from which the $\mathbf{H}$ deflection was recor- 

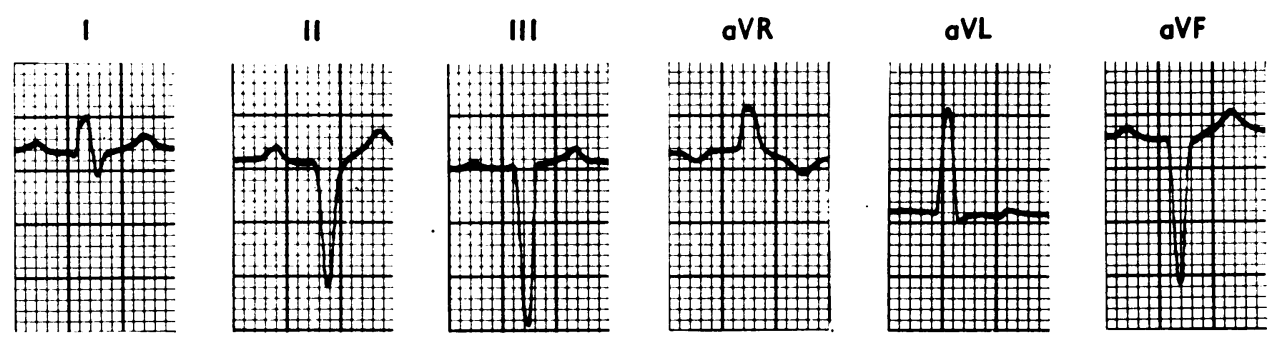

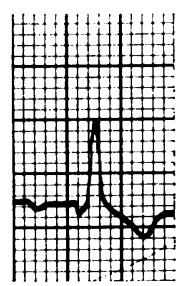

VI

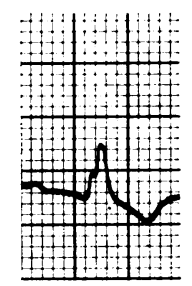

V2

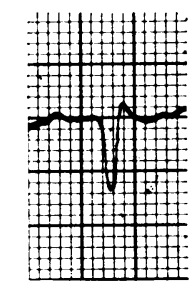

V3

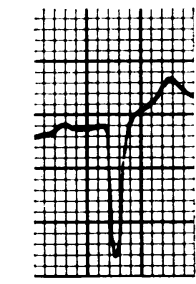

V4

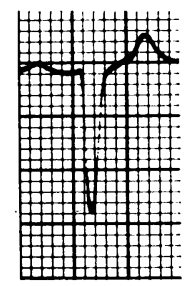

V5

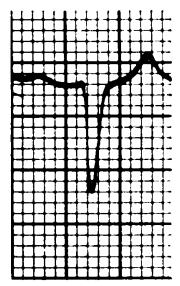

V6

FIG. I Twelve-lead electrocardiogram during sinus rhythm showing right bundle-branch block, left anterior hemiblock, and possibly old anterior and inferior wall myocardial infarctions. The PR interval measured $180 \mathrm{~ms}$.

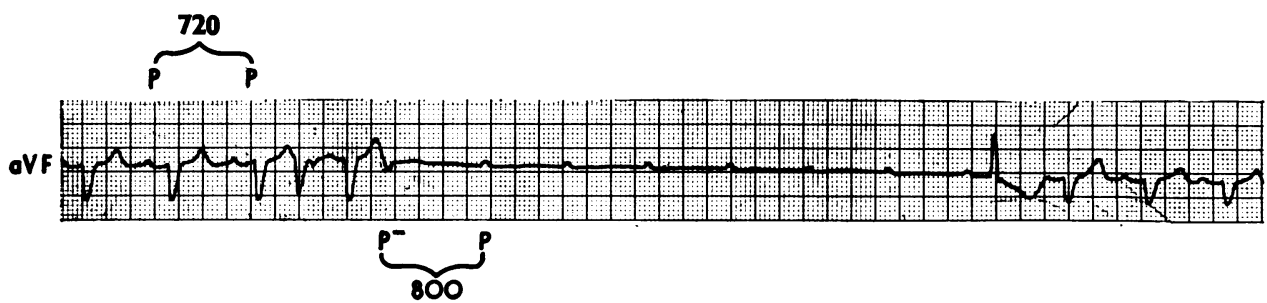

FIG. 2 Bradycardia-dependent atrioventricular block triggered by premature beats (fourth and fifth $Q R S$ complexes). Whereas the intervals between two consecutive sinus $P$ waves measured $720 \mathrm{~ms}$ that between $P^{-}$and the subsequent sinus $P$ wave measured $800 \mathrm{~ms}$. In this and the other illustration the intervals are expressed in ms.

ded) or in the left bundle-branch or its posteroinferior division. The duration of the remaining conduction intervals was normal.

Premature atrial stimuli were delivered at varying coupling intervals to study the effects of post-ectopic cycle length changes. Fig. 4 shows that the control PP and $\mathrm{HH}$ intervals measured $730 \mathrm{~ms}$. The third (premature) atrial impulse $\left(\mathbf{P}^{+}\right)$arriving at the His bundle $325 \mathrm{~ms}$ after the preceding $\mathrm{H}$ deflection failed to activate the ventricles. It also prolonged the corresponding $P^{+}-\mathbf{P}$ and $\mathrm{HH}$ intervals to $875 \mathrm{~ms}$, thereby initiating a run of atrioventricular block during which the supraventricular impulses were blocked below the site from which the $\mathbf{H}$ deflection was recorded (Fig. 4). This arrhythmia lasted until a ventricular escape beat reinitiated I:I atrioventricular conduction (Fig. 5).

The effects of atrial pacing at increasing rates $(150 / \mathrm{min}$ at the beginning and 200/min towards the end of Fig. 6) were also studied. This procedure resulted in a gradual prolongation of the $\mathrm{AH}$ intervals and in a decrease in the corresponding $\mathrm{HH}$ intervals. Block below the site from which the $\mathrm{H}$ deflection was recorded occurred when the $\mathrm{HH}$ interval was reduced to a value of $320 \mathrm{~ms}$.

It was concluded that this patient had symptomatic bradycardia-dependent atrioventricular block. Moreover, tachycardia-dependent atrioventricular block was apparently also present. The latter conduction disturbance should be related to an increase in rate (Fig. 6) or to a 


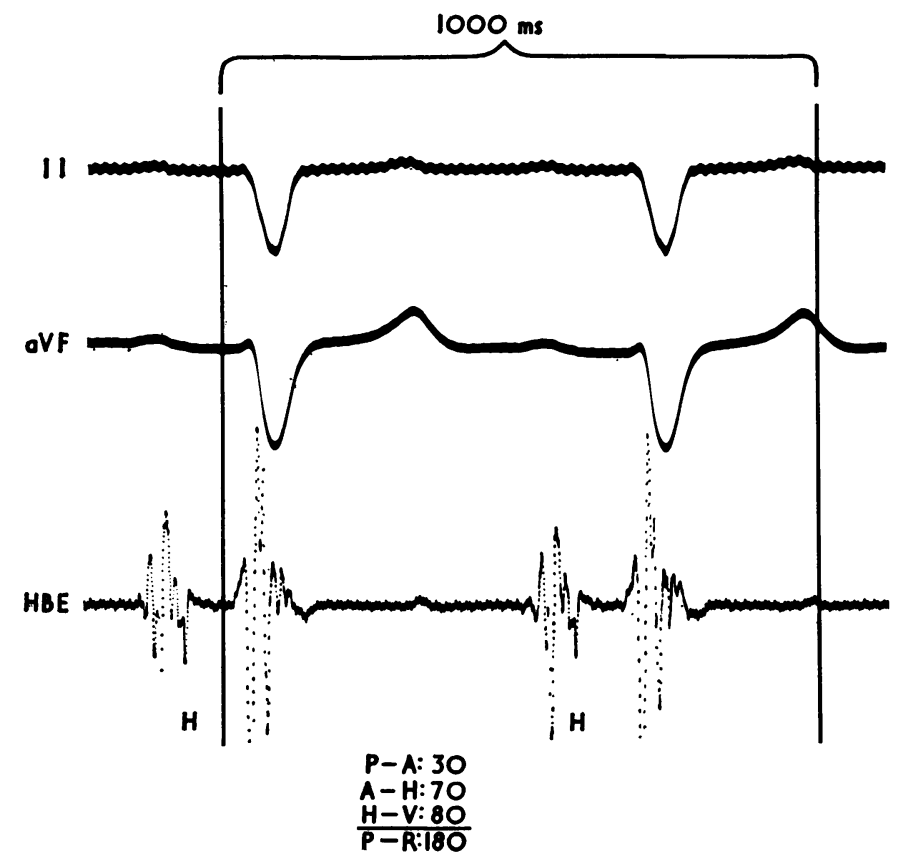

FIG. 3 His bundle electrograms recorded during sinus rhythm showing prolongation of the HV interval. Abbreviations are conventional. In this and subsequent illustrations paper speed and intervals between time marks were $100 \mathrm{~mm} / \mathrm{s}$ and I $\mathrm{s}$, respectively.

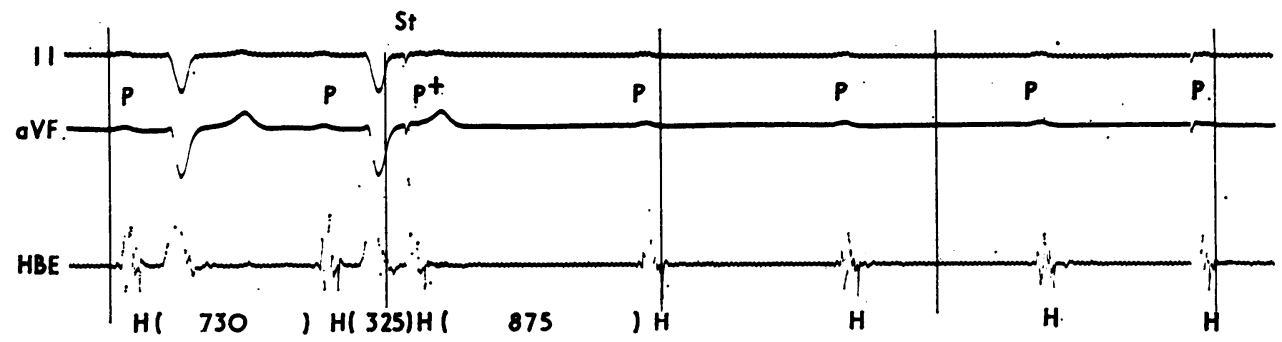

FIG. 4 Episode of bradycardia-dependent atrioventricular block triggered by a single (electrical) premature atrial impulse $\left(P^{+}\right)$blocked below the site from which the $H$ deflection was recorded. Note the resemblance between the electrically-induced events in this Figure and those appearing spontaneously in Fig. 2. Non-conducted $P$ waves were also blocked below the $H$ deflection. Infra-His bundle block at an HH interval of $325 \mathrm{~ms}$ is probably an expression of tachycardiadependent atrioventricular block.

decrease in cycle length (Fig. 4) and not exclusively to a faster rate. Both forms of atrioventricular block could have occurred in the low His bundle (below the site from which the $\mathrm{H}$ deflection was recorded), or in the left bundle-branch or in its posteroinferior division.

The patient was referred to the Division of Cardiovascular Surgery for pacemaker implantation. 


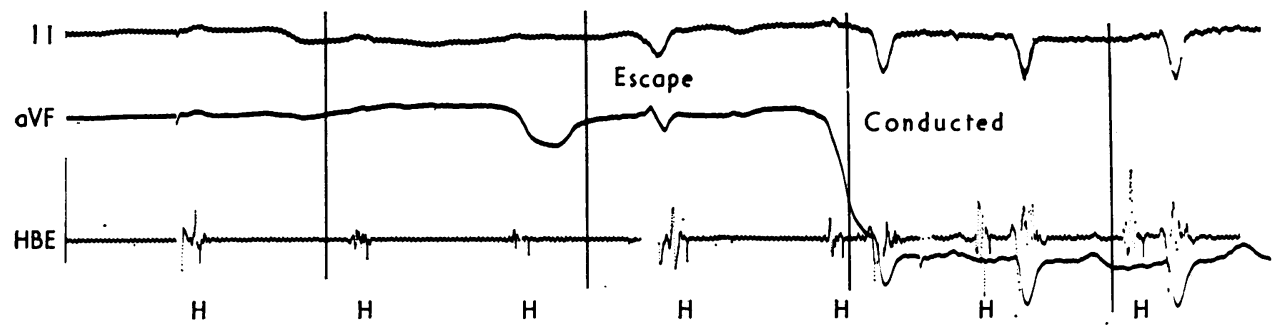

FIG. 5 End of episode of bradycardia-dependent atrioventricular block after a ventricular escape beat. The latter was (as in Fig. 2) followed by resumption of $1: 1$ atrioventricular conduction.

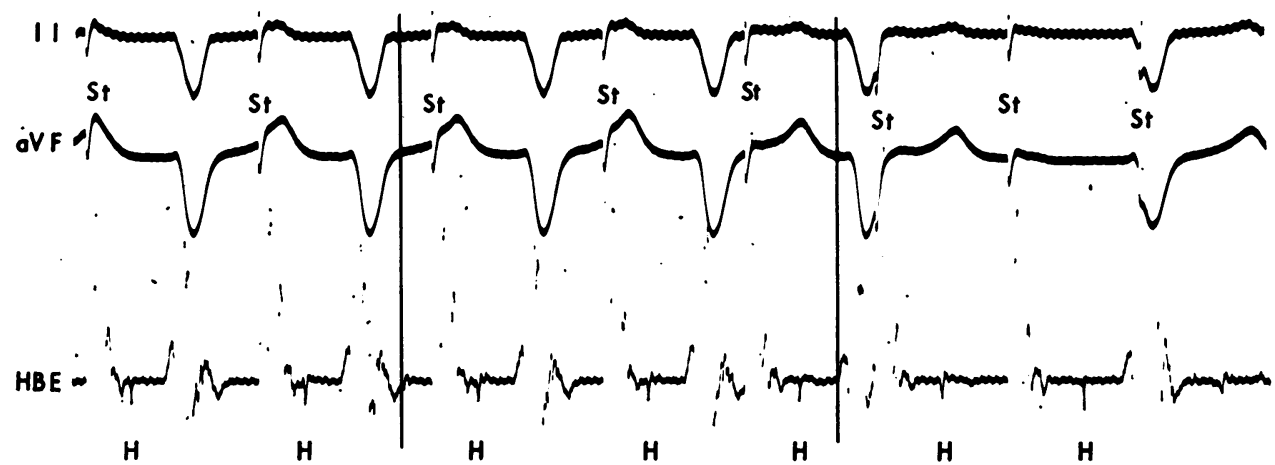

FIG. 6 Block below the $H$ deflection appearing during atrial stimulation at progressively higher rates. The conduction failure occurred when the HH interval was reduced to a value of $320 \mathrm{~ms}$.

spontaneously, but in some cases (Fig. 2 and 4 ) it is produced by the post-extrasystolic pause after atrial or atrioventricular junctional and ventricular extrasystoles with retrograde conduction (Coumel et al., I97I ; Rosenbaum et al., 1971).

However, the important factor is not the decrease in sinus rate per se but the resulting reduction of the corresponding $\mathrm{HH}$ intervals. Because of the latter, the impulse reaches a group of cells having an enhanced or slightly rising slope of phase 4 (diastolic) depolarization. This gradual shift of the membrane potential towards zero determines the conduction failure of impulses arriving relatively late in the cycle. Earlier impulses can propagate if the membrane potential is farther away from zero (closer to $-90 \mathrm{mV}$ ).

In addition to phase 4 depolarization some authors believe that a decrease in membrane responsiveness can contribute to the occurrence of bradycardiadependent atrioventricular block (El-Sherif et al., 1974; Rosenbaum et al., 1974). This interesting assumption requires further evaluation.
It should be emphasized that the existence of phase 4 diastolic depolarization does not necessarily imply that automaticity is increased. For instance, escapes from the affected area do not occur frequently, indicating the existence of a gradual (definitely abnormal) shift of the threshold potential towards zero (El-Sherif et al., r974; Rosenbaum et al., 1974). Thus, automaticity is probably depressed rather than enhanced in these cases.

According to El-Sherif et al. (1974) the relatively long intervals usually required for bradycardiadependent atrioventricular block to occur favour a normal or slightly rising (rather than an enhanced) slope of phase 4 depolarization.

Fig. 4 suggests that after the onset of bradycardiadependent atrioventricular block membrane potential remained at a value that did not allow conduction beyond the affected area, regardless of the duration of subsequent HH (or PP) intervals. Cycle length, therefore, plays an important role only in regard to the initiation, not the perpetuation, of the arrhythmia. 
Spontaneous escapes were not present in the record shown in Fig. 4 and atrioventricular block persisted until the appearance later of an escape beat (Fig. 5). Rosenbaum et al. (1973a) have suggested that in bradycardia-dependent atrioventricular block, the block is unidirectional since retrograde activation of the affected area is preserved. These authors believe that ventricular escapes, no matter where they originate, most probably penetrate the diseased region retrogradely and bring back (reset) the membrane potential towards its maximal (more normal) value. Consequently the oncoming supraventricular impulse is able to be propagated and atrioventricular conduction starts anew.

Bradycardia-dependent atrioventricular block seems to occur only in tissues showing phase 4 depolarization. Therefore, the affected area could be located in the His bundle, bundle-branches, or divisions of the left bundle-branch. The involved structure must be the only available connexion between atria and ventricles for otherwise only isolated bradycardia-dependent bundle-branch block or divisional block would result.

As a rule the phenomenon under consideration is seen in patients with bundle-branch block suggesting that it is caused by an abnormality located in the non-involved branch or division. In the case reported by Coumel et al. (197I) (with narrow QRS), the 'low' His bundle appeared as the most likely site of block. Wide ventricular complexes do not exclude intra-His bundle block even if (as in the patient discussed in this report) the HV interval is prolonged since the affected region could have been located in the low His bundle, in the left bundlebranch, or in its posteroinferior division. Two cases in which the conduction disturbance seemed to have occurred in the 'upper' His bundle will be discussed subsequently.

Fig. 4 and 6 suggest that tachycardia-dependent atrioventricular block was also present, since block was seen whenever the $\mathrm{HH}$ intervals were reduced below a critical value either by premature atrial stimulation or rapid atrial pacing. The tachycardiadependent atrioventricular block could be related to either prolonged duration of the action potential (voltage-dependent factor) (Rosenbaum et al., 1973a) or to a time-dependent response in the affected cells (El-Sherif et al., I974).

Fig. 4 and 6 show that atrioventricular block was seen at both relatively long and short cycle lengths with an intermediate normal conduction range. Moreover, the prolonged HV interval in conducted beats indicated the coexistence of depressed conduction velocity in the affected region. Whether a prolonged HV interval is present in all cases of bradycardia-dependent atrioventricular block is not known. Nevertheless, it is suggested that all patients with prolonged HV intervals in whom bradycardiadependent atrioventricular block does not occur spontaneously should be studied with this possibility in mind.

There are several ways to induce this phenomenon. Carotid sinus massage can produce bradycardia-dependent atrioventricular block within the His-Purkinje system by simply prolonging the $\mathrm{PP}$ and $\mathrm{HH}$ intervals. But vagal stimulation also causes atrioventricular nodal block. Therefore, this manoeuvre introduces additional problems of interpretation.

This was evident in two cases recently reported in which the conduction defect presumably occurred within the His bundle, above the site from which the H deflection was recorded (Castellanos et al., 1974; Goodfriend and Barold, 1974). These patients are interesting because of atrial electrograms not being followed by $\mathrm{H}$ deflections. Moreover retrograde activation of the His bundle did not abolish the paroxysms of atrioventricular block. In fact, atrioventricular conduction was reinitiated spontaneously, not by an escape as in our Fig. 2 and 6. Though bradycardia-dependent atrioventricular block at the upper His bundle was a distinct possibility, vagal-block at the atrioventricular node or $\mathrm{N}-\mathrm{H}$ region could not be excluded in these cases. The type II (Mobitz) pattern observed by the authors might have resulted from the moment in the cycle (after the end of the PR interval) in which the vagal effects first became manifested.

Another method used to induce bradycardiadependent atrioventricular block is the introduction of properly timed single (Fig. 4) or multiple atrial impulses, a procedure that can result in considerable lengthening of the post-extrasystolic $\mathrm{HH}$ interval (see Fig. 5 of the article by Agha et al., 1973).

Finally, the events seen in this case emphasize the importance of the procedure of artificial induction of arrhythmias in corroborating the mechanisms of complex arrhythmias derived from the analysis of surface electrocardiograms.

\section{References}

Agha, A. S., Castellanos, A., Jr., Wells, D., Ross, M. D., Befeler, B., and Myerburg, R. J. (I973). Type I, Type II, and Type III gaps in bundle-branch conduction. Circulation, 47, 325.

Castellanos, A., Sung, R. J., Cunha, D., and Myerburg, R. J. (1974). His bundle recordings in paroxysmal atrioventricular block produced by carotid sinus massage. British Heart fournal, 36, 487.

Castillo, C. A., and Castellanos, A., Jr. (1971). Retrograde activation of the His bundle in the human heart. American Fournal of Cardiology, 27, 264. 
Corrado, G., Levi, R. J., Nau, G. J., and Rosenbaum, M. B. (1974). Paroxysmal atrioventricular block related to phase 4 bilateral bundle branch block. American fournal of Cardiology, 33, 553.

Coumel, P., Fabiato, A., Waynberger, W., Motte, G., Slama, R., and Bouvrain, Y. (197I). Bradycardia-dependent atrioventricular block. Report of two cases of A-V block elicited by premature beats. Fournal of Electrocardiology, 4, 168 .

Elizari, M. V., Nau, G. J., Levi, R. J., Lazzari, J. O., Halpern, M. S., and Rosenbaum, M. B. (1974). Experimental production of rate-dependent bundle branch block in the canine heart. Circulation Research, 34, 730.

El-Sherif, N., Scherlag, B. J., Lazzara, R., and Samet, P. (1974). Pathophysiology of tachycardia- and bradycardiadependent block in the canine proximal His-Purkinje system after acute myocardial ischemia. American fournal of Cardiology, 33, 529.

Goodfriend, M. A., and Barold, S. S. (1974). Tachycardiadependent and bradycardia-dependent Mobitz Type II atrioventricular block within the bundle of His. American fournal of Cardiology, 33, 908.
Rosenbaum, M. S., Elizari, M. V., Chiale, P., Levi, R. J., Nau, G. J., Halpern, M. S., Lazzari, J. O., and Novakowsky, A. (1974). Relationships between increased automaticity and depressed conduction in the main intraventricular conducting fascicles of the human and canine heart. Circulation, $49,818$.

Rosenbaum, M. B., Elizari, M. V., Lazzari, J. O., Halpern, M. S., Nau, G. J., and Levi, R. J. (1973a). The mechanism of intermittent bundle branch block. Relationship to prolonged recovery, hypopolarization and spontaneous diastolic depolarization. Chest, 63, 666.

Rosenbaum, M. B., Elizari, M. V., Levi, R. J., and Nau, G. J. (1973b). Paroxysmal atrioventricular block related to hypopolarization and spontaneous diastolic depolarization. Chest, 63, 678.

Requests for reprints to Professor Agustin Castellanos, University of Miami School of Medicine, P.O. Box 520875, Biscayne Annex, Miami, Florida 33152, U.S.A. 